\title{
Reclassifying ovarian cancer: origins, subtypes and resistance to therapy
}

\author{
DDL Bowtell \\ From Familial Aspects of Cancer 2011 Research and Practice: A combined meeting of kConFab, Australian \\ Breast Cancer Family Study, Australian Colorectal Cancer Family Study, Australian Ovarian Cancer Study, \\ Family Cancer Clinics of Australia and New Zealand and kConFab \\ Kingscliff, Australia. 23-26 August 2011
}

Recent pathological and molecular studies have forced a very significant re-evaluation of the conventional classification of EOC. Microarray and other molecular experiments demonstrate that EOC is a series of molecularly distinct diseases that individually bear more resemblance to certain non-ovarian cancers than they do to each other. Ovarian cancer really represents a spectrum of distinct diseases that share an anatomical location. The presentation focuses on the increasing understanding of the molecular differences between and within different ovarian cancer histotypes. Particular attention is given to high-grade ovarian serous cancers, which account for about two thirds of ovarian cancer deaths, and ovarian clear cell cancers, a tumour type with generally poor response to platinum-based therapy.

Using both gene expression (GE) and DNA copy number $(\mathrm{CN})$ analyses, we have defined novel molecular subtypes of high-grade serous cancers [1]. The molecular subtypes are robustly represented in multiple datasets and are associated with distinct clinical outcomes, and therefore appear to be biologically meaningful. Our efforts to understand the drivers of molecular subtypes of high-grade serous will be discussed [2,3]. A clear cell cohort was analysed using GE and $\mathrm{CN}$ analyses, demonstrating deregulation of receptor tyrosine kinases and cytokine pathways [4]. In particular, deregulation of IL6/ STAT3/HIF pathway and its targeting in a clinical setting will be described.

Platinum remains the mainstay of treatment for highgrade serous cancers, however, about $20 \%$ of patients fail initial treatment and of those that respond, the majority relapse within 2 years and progressively develop resistance to treatment. We have identified mechanisms of primary treatment failure $[5,6]$ and are currently analysing paired primary and relapse samples to determine mechanisms of acquired treatment failure as part of the ICGC project. Studies in treatment resistance will be discussed.

\section{Published: 12 April 2012}

\section{References}

1. Tothill RW, et al: Novel molecular subtypes of serous and endometrioid ovarian cancer linked to clinical outcome. Clin Cancer Res 2008, 14:5198-5208.

2. Bowtell DD: The genesis and evolution of high-grade serous ovarian cancer. Nat Rev Cancer 2010, 10:803-808.

3. Helland A, et al: Deregulation of MYCN, LIN28B and LET7 in a molecular subtype of aggressive high-grade serous ovarian cancers. PLoS One 2011, 6:e18064.

4. Anglesio MS, et al: IL6-STAT3-HIF signaling and therapeutic response to the angiogenesis inhibitor sunitinib in ovarian clear cell cancer. Clin Cancer Res 2011, 17:2538-2548.

5. Etemadmoghadam D, et al: Integrated genome-wide DNA copy number and expression analysis identifies distinct mechanisms of primary chemoresistance in ovarian carcinomas. Clin Cancer Res 2009, 15:1417-1427.

6. Etemadmoghadam D, et al: Amplicon-dependent CCNE1 expression is critical for clonogenic survival after cisplatin treatment and is correlated with 20q11 gain in ovarian cancer. PLoS One 2010, 5:e15498.

doi:10.1186/1897-4287-10-S2-A35

Cite this article as: Bowtell: Reclassifying ovarian cancer: origins, subtypes and resistance to therapy. Hereditary Cancer in Clinical Practice 2012 10(Suppl 2):A35. 